\title{
Characterizing Multivalent Metal Anodes with Cryogenic Electron Microscopy
}

\author{
Daniel Long ${ }^{1}$, Scott McClary ${ }^{2}$, Paul Kotula ${ }^{1}$, Kevin Zavadil $^{2}$ and Katherine Jungjohann ${ }^{2}$ \\ ${ }^{1}$ Sandia National Laboratories, Albuquerque, New Mexico, United States, ${ }^{2}$ Sandia National Laboratories, \\ United States
}

The decarbonization of our energy ecosystem, including grid-scale storage and electrification of passenger vehicles, necessitates the development of higher energy density batteries. Lithium metal batteries are a promising candidate for the transition to a more battery-laden energy ecosystem; ${ }^{1}$ however, the low natural abundance of lithium requires that long-term solutions utilize other chemistries. ${ }^{2}$ Calcium and magnesium exhibit high natural abundance, divalency, low mass, high density, and the ability to reversibly electrodeposit, making them suitable anodes for rechargeable multivalent metal batteries (RMVMB). Successful RMVMB development is dependent on improving the energy density and Coulombic efficiency of metal deposition, which are governed by metal microstructural evolution and the solid electrolyte interphase (SEI) formed in candidate liquid electrolytes. These two anode attributes need to be conclusively defined for RMVMBs anode development.

Cryogenic electron microscopy is a valuable tool to investigate SEI structure and chemistry because of its ability to preserve liquid/solid interfaces, stabilize delicate phases, and reduce beam damage and atmospheric contamination. Here we utilize cryogenic-FIB lamella preparation and various cryo-TEM techniques to characterize the structure and chemistry of dense $\mathrm{Ca}$ and $\mathrm{Mg}$ deposits formed from select electrolytes. Sample preparation was performed with a commercial Leica cryogenic workflow cooled to at least $-150{ }^{\circ} \mathrm{C}$, and cryoFIB was performed following published cryogenic and atomic resolution guides. ${ }^{3-5}$ After metal anode deposition anhydrous diglyme $(\mathrm{G} 2)$ was used to displace the volatile THF-based electrolytes used during deposition in order to stabilize the SEI for transport. Excess G2 was first wicked from the deposit and then further trimmed down via a grazing incidence mill with the $\mathrm{Ga}^{+}$ion beam. Careful thinning with small sample tilts and low energy ion beams allows for reproducible lamella thicknesses below $100 \mathrm{~nm}$. TEM investigation was performed with an FEI Titan G2 80-200 operated at $200 \mathrm{kV}$ with a Gatan Quantum 963 EELS/imaging filter system.

The lifted-out sample shown in the SEM micrograph in Figure 1a has a dense and flat Ca deposit with a thin intact G2 cap. The brightfield TEM micrograph in Figure 1b shows the fully dense Ca deposit on a Au electrode with a $10 \mathrm{~nm} \mathrm{Au} / \mathrm{Ca}$ intermetallic phase. This mixed phase is easily altered by the $\mathrm{Ga}^{+}$ion beam and its true characteristics are only properly captured with cryogenic FIB preparation. The contrast in the $\mathrm{Ca}$ in the brightfield micrograph and the streaking in the bulk Ca SAD pattern in Figure 1c indicate that stacking faults are prevalent in the Ca deposit. Discontinuous diffraction rings in the diffraction pattern (Figure 1c) taken from the middle of the deposit indicate that the $\mathrm{Ca}$ film is heavily textured. Indexing the diffraction pattern determines the presence of both the stable Ca FCC phase as well as the less energetically stable $\mathrm{Ca} \mathrm{HCP}$ phase. A diffuse $\mathrm{CaO}$ reflection is also present and increases with illumination time due to Ca oxidation by reaction with trace ice contamination.

Electron energy loss spectroscopy was used to determine the chemical content of the Ca metal anode and its SEI. The Ca SEI region of interest is shown in the STEM brightfield micrograph in Figure 2a along with chemical component images formed from multivariate statistical analysis of core-loss EELS spectral images. ${ }^{6}$ Four unique curves were resolved: 1) $\mathrm{Ca}$ in the $\mathrm{Ca}$ deposit(not shown), 2) $\mathrm{C}$ and $\mathrm{O}$ in the G2(not shown), 3) $\mathrm{B}, \mathrm{C}, \mathrm{Ca}, \mathrm{O}$ in the SEI and G2 (Figure 2b), and 4) Ca, O in the SEI and G2(Figure 2c). The strong Ca, O curve in the SEI is expected as prior work ${ }^{7}$ has shown that a nanoscale oxide is present at the Ca surface. Boron in the SEI has been found in other borate-containing electrolytes ${ }^{8}$ and is under further investigation. Extensive detail about the structure and chemistry of the bulk, SEI, and co-depositing hydride phases will be presented. 


\section{Acknowledgements}

This work was supported by the Joint Center for Energy Storage Research, an Energy Innovation Hub funded by the U.S. Department of Energy. This work was performed, in part, at the Center for Integrated Nanotechnologies, an Office of Science User Facility operated for the U.S. Department of Energy (DOE) Office of Science. Sandia National Laboratories is a multimission laboratory managed and operated by National Technology \& Engineering Solutions of Sandia, LLC, a wholly owned subsidiary of Honeywell International, Inc., for the U.S. DOE's National Nuclear Security Administration under contract DE-NA0003525. The views expressed in the article do not necessarily represent the views of the U.S. DOE or the United States Government. Unclassified unlimited release, SAND2021-2223 A.
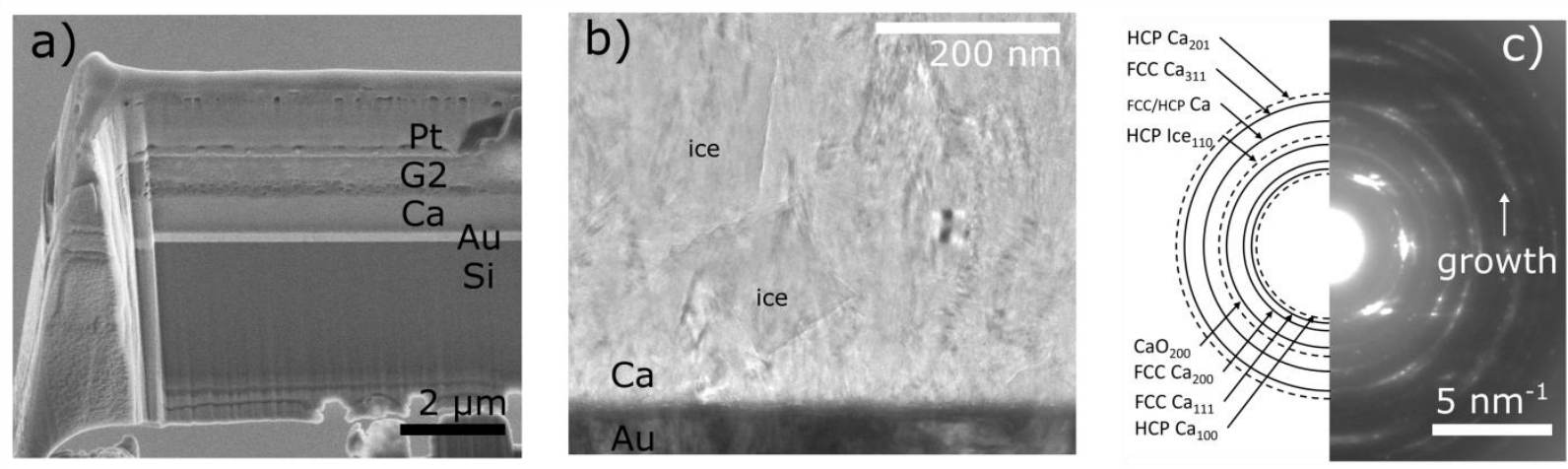

Figure 1. Electron transparent lamella with thin electrolyte caps are reproducibly created with the Leica cryogenic workflow and careful cryo-FIB preparation. (a)The SEM micrograph reveals the dense and planar nature of the Ca deposit and the (b)BF TEM micrograph illuminates the interfacial structure and strain in the deposit. (c)Diffraction patterns reveal both the FCC and HCP phase with extensive texturing within the growth plane.
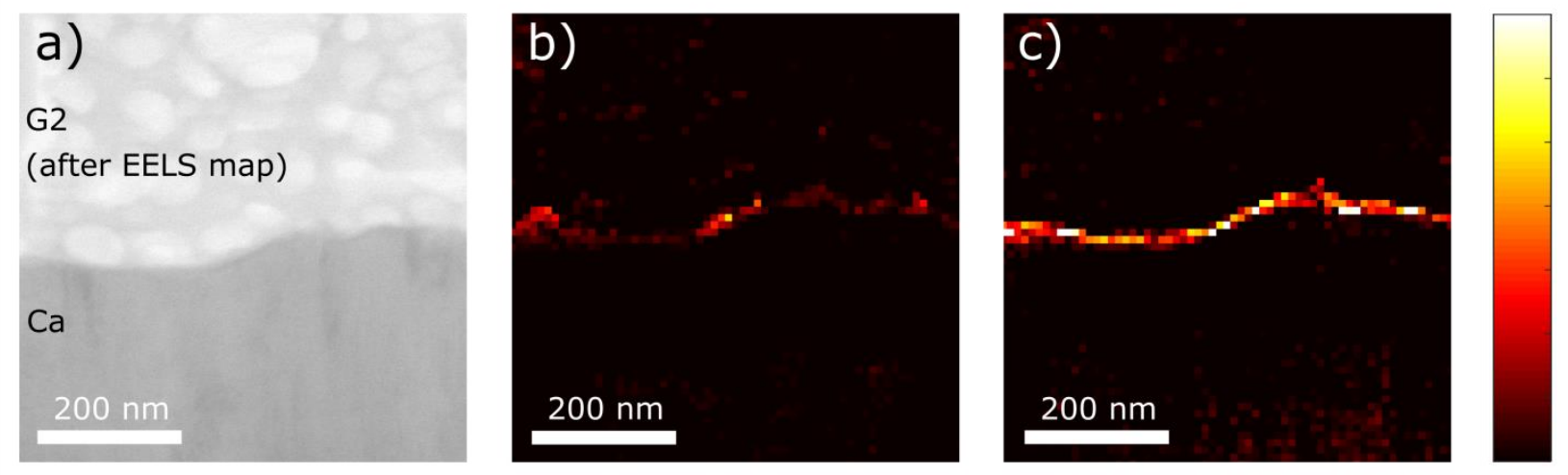

Figure 2. The investigated region shown in the (a) STEM BF micrograph contains $C a$, its interphase, and the G2 cap. EELS maps of the coreloss show the (b) local boron and (c) oxygen concentrations respectively.

\section{References}

1. Harrison, K. L.; Zavadil, K. R.; Hahn, N. T.; Meng, X.; Elam, J. W.; Leenheer, A.; Zhang, J. G.; Jungjohann, K. L., Lithium Self-Discharge and Its Prevention: Direct Visualization through in Situ Electrochemical Scanning Transmission Electron Microscopy. ACS Nano 2017, 11, 11194-11205.

2. Ponrouch, A.; Bitenc, J.; Dominko, R.; Lindahl, N.; Johansson, P.; Palacin, M. R., Multivalent Rechargeable Batteries. Energy Storage Materials 2019, 20, 253-262. 
3. Zachman, M. J.; Asenath-Smith, E.; Estroff, L. A.; Kourkoutis, L. F., Site-Specific Preparation of Intact Solid-Liquid Interfaces by Label-Free in Situ Localization and Cryo-Focused Ion Beam Lift-Out. Microsc Microanal 2016, 22, 1338-1349.

4. Rubino, S.; Akhtar, S.; Melin, P.; Searle, A.; Spellward, P.; Leifer, K., A Site-Specific Focused-Ion-Beam Lift-out Method for Cryo Transmission Electron Microscopy. J Struct Biol 2012, 180, 572-6.

5. Schaffer, M.; Schaffer, B.; Ramasse, Q., Sample Preparation for Atomic-Resolution Stem at Low Voltages by Fib. Ultramicroscopy 2012, 114, 62-71.

6. Sugar, J. D.; El Gabaly, F.; Chueh, W. C.; Fenton, K. R.; Tyliszczak, T.; Kotula, P. G.; Bartelt, N. C., High-Resolution Chemical Analysis on Cycled Lifepo4 Battery Electrodes Using Energy-Filtered Transmission Electron Microscopy. Journal of Power Sources 2014, 246, 512-521.

7. Long, D.; McClary, S.; Hahn, N.; Zavadil, K.; Jungjohann, K., Chemistry, Microstructure, and Interphases of Magnesium Metal Anodes Captured Via Cryogenic Electron Microscopy. ECS Meeting Abstracts 2020, MA2020-02, 776-776.

8. Forero-Saboya, J.; Davoisne, C.; Dedryvère, R.; Yousef, I.; Canepa, P.; Ponrouch, A., Understanding the Nature of the Passivation Layer Enabling Reversible Calcium Plating. Energy \& Environmental Science 2020. 